\title{
Octogenários: resultados de 3659 necropsias (12 anos de observação)
}

\author{
José Carlos R. IGLÉZIAS*, Artur LOURENÇĀO Jr., Luís Alberto DALLAN*, Paulo Sampaio GUTIERRES*, \\ José Antônio F. RAMIRES*, Noedir A. G. STOLF*, Sérgio Almeida de OLIVEIRA*, Geral̄̄o VERGINELLI*', \\ Adib D. JATENE*
}

IGLÉZIAS, J. C. R.; LOURENÇĀO Jr., A.; DALLAN, L. A.; GUTIERRES, P. S.; RAMIRES, J. A. F.; STOLF, N. A. G.; OLIVEIRA, S. A.; VERGINELLI, G.; JATENE, A. D. - Octogenários: resultados de 3659 necropsias (12 anos de observaçāo). Rev. Bras. Cir. Cardiovasc., 8(1):39-43, 1993.

RESUMO: No InCór, no período compreendido entre janeiro de 1978 e dezembro de 1990, foram realizadas 3659 necropsias, das quais 106 relativas a pacientes com idade $\geqslant 80$ anos. Destes, $55(51,88 \%)$ eram do sexo masculino e $51(48,11 \%)$ do feminino. A média de idade foi igual a 83,66 ( 80 a 93 anos). Dentre os principais sintomas (Gráfico 3 ) apresentados figuravam: a angima do peito em $40,56 \%$, a dispnéia em $25,47 \%$ e a síncope em 8,49 deles. Em $47,16 \%$ dos pacientes os sintomas haviam se iniciado a mais de seis meses. Dentre os sistemas desencadeantes dos óbitos (Gráfico 1), encontramos o cardiovascular em $74,52 \%$, o respiratório em $10,37 \%$, o digestivo em $9,43 \%$, o nervoso em $1,88 \%$, o urinário em $1,88 \%$ e outros em 1,88\%. Entre os óbitos cardiovasculares (Gráfico 2), $56,96 \%$ foram devidos a coronariopatia, $12,65 \%$ a miocardiopatia, $10,12 \%$ devido aos aneurismas da aorta e $8,86 \%$ atribuídos às arritmias. Baseados na casuística prévia e considerando: 1) que o sistema cardiovascular foi o maior determinante dos óbitos $(74,52 \%) ; 2$ ) que as coronariopatias foram responsáveis por $56,96 \%$ dos óbitos cardiovasculares; 3 ) que a revascularizaçāo convencional neste grupo tem mortalidade atual de $8,5 \%$; 4 ) que a angioplastia primária foi bem sucedida em $93,2 \%$, concluímos que ambas, a qualidade e a expectativa de vida, podem ser melhoradas trazendo o octogenário para próximo do médico, ou reduzindo o intervalo entre suas avaliaçōes.

DESCRITORES: necropsias, pacientes idosos.

\section{INTRODUÇĀO}

Artigos médicos publicados em 1989 expressaram que a doença coronária seria moléstia da pessoa idosa.

Recentemente, ROBERTS et alii ${ }^{10}$ relataram pacientes acometidos por doença arterial coronária fatal e que foram submetidos a estudo necroscópico. Dos 867 pacientes com idade superior a 30 anos, acometidos por doença arterial coronária fatal que não haviam sido previamente revascularizados ou submetidos a angioplastia ou outro tipo de operaçāo cardíaca, classificaram o coração de acordo com o evento final em quatro tipos de óbitos: infarto agudo do miocárdio, morte súbita fora do ambiente hospitalar, insuficiência cardíaca congestiva após cicatrizaçāo de infarto agudo do miocárdio e morte súbita hospitalar nos portadores de angina instável. A média de idade dos 667 homens foi de 60 anos e das mulheres, 68 anos. Trezentos e noventa e três ( $45 \%)$, dos 867 pacientes, faleceram com idade variando entre 31 e 60 anos, sendo $343(87 \%)$ homens e 50 $(13 \%)$ mulheres. A porcentagem de mulheres aumentou progressivamente nas três últimas décadas consideradas: 1961 a 1970,1971 a 1980 e 1981 a 1990. Dos 256 pacientes com idade entre 61 e 70 anos, $53(21 \%)$ eram mulheres e $203(79 \%)$ eram homens. Dos 161 pacientes com idade variando

Trabalho realizado no Instituto do Coração do Hospital das Clínicas da Faculdade de Medicina da Universidade de São Paulo. São Paulo, SP, Brasil. Recebido para publicação em 28 de março de 1993.

* Do Instituto do Coração do Hospital das Clínicas da Faculdade de Medicina da Universidade de São Paulo.

Endereço para separatas: José Carlos Iglésias. Av. Dr. Enéas de Carvalho Aguiar, 44. Divisão Cirúrgica. 05403 Săo Paulo, SP, Brasil. 
IGLÉZIAS, J. C. R.; LOURENÇÄO Jr., A.; DALLAN, L. A.; GUTIERRES, P. S.; RAMIRES, J. A. F.; STOLF, N. A. G.; OLIVEIRA, S. A.; VERGINELLI, G.; JATENE, A. D. - Octogenários: resultados de 3659 necropsias (12 anos de observação). Rev. Bras. Cir. Cardiovasc., 8(1):39-43, 1993.

entre 72 e 80 anos, $65(40 \%)$ eram mulheres e 96 $(60 \%)$ eram homens e dos 51 pacientes restantes com idade variando entre 81 a 90 anos, 28 (55\%) eram mulheres e $23(45 \%)$ eram homens. Do grupo todo, $667(77 \%)$ eram homens e $200(23 \%)$ eram mulheres. $\mathrm{O}$ grupo de morte coronária súbita apresentou um número significante $(p<0,05)$ de homens mais jovens do que os outros três grupos; a média de idade do grupo com infarto agudo do miocárdio foi significantemente $(p<0,05)$ mais elevada (65 anos) do que a dos outros três grupos. Os grupos com insuficiência cardíaca congestiva e angina do peito instável tinham média de idade semelhante (62 e 63 anos), e intermediária. Os grupos de morte coronária súbita e $\circ$ de insuficiência cardíaca congestiva crônica apresentavam maior porcentagem de homens $(90 \%$ a $86 \%$, respectivamente). Nos grupos do infarto agudo do miocárdio e da angina do peito instável, a porcentagem de homens foi menor $(66 \%$ e $73 \%)$. Nenhuma mudança significativa foi notada nas três décadas, em cada gênero, com cada um dos quatro grupos.

ARONOW et alii ${ }^{1}$, tentando analisar o prognóstico para o paciente idoso com e sem prejuízo da função sistólica ventricular esquerda associada a doença arterial coronária e insuficiência cardíaca congestiva, afirmam, baseados em dados de seu estudo prospectivo, que a fração de ejeção do ventrículo esquerdo (FEVE) representa a variável mais importante no paciente idoso. As taxas de sobrevida para pacientes portadores de insuficiência cardíaca associada a doença arterial coronária e FEVE normal foi de $78 \%$ no $1^{\circ}$ ano, de $62 \%$ no $2^{\circ}$ ano, $54 \%$ no $3^{\circ}$ ano e $44 \%$ no $4^{\circ}$ ano. As taxas de sobrevida para os pacientes portadores de insuficiência cardíaca congestiva (ICC) associada a insuficiência coronária e FEVE anormal foi de $53 \%$ no $1^{2}$ ano, $29 \%$ no $2^{\circ}$ ano, $22 \%$ no $3^{\circ}$ ano e $15 \%$ no $4^{\circ}$ ano.

MACAYA et alii ${ }^{7}$, analisando os resultados e complicaçōes no seguimento tardio, clínico e angiográfico, da angioplastia em pacientes com idade > 65 anos, e comparando os dados com os da população mais jovem correlacionada, relatam sucesso primário de $88 \%$ naqueles com idade $>65$ anos e $89 \%$ nos com idade $<65$ anos.

MULLANY et alii ${ }^{8}$, analisando 159 pacientes com idade $>80$ anos, submetidos a revascularização do miocárdio na Mayo Clinic, desde 1977, concluíram que a operação em pacientes dessa idade, embora associada com risco operatório aumentado, propicia um excelente alívio dos sintomas e boa sobrevida de cinco anos, e que os mesmos não podem ser desconsiderados para a revascularizaçāo do miocárdio somente devido à idade.
LOOP et alii ${ }^{6}$, analisando 5070 pacientes com idade > 65 anos, submetidos a revascularização eletiva do miocárdio, subdivididos em subgrupos de 65 a 74 anos e de 75 anos em diante, quando comparados com pacientes mais jovens (idade $<65$ anos) concluíram que, com o avançar da idade, existe grande prevalência de mulheres, angina do peito mais severa, presença de diabetes, doença vascular periférica, eventos cerebrais prévios, aumento do índice cardiotorácico e doença do tronco coronário esquerdo. Observaram que a mortalidade global durante a hospitalização foi de $2,3 \%$ e que a mesma subiu progressivamente com o avanço da idade. Dentre os fatores previsores para a elevada mortalidade hospitalar, figuravam: idade igual ou superior a 75 anos, tabagismo, prejuízo da função ventricular esquerda e sexo feminino. A sobrevida de dez anos foi de $64 \%$ para os pacientes com idade entre 65 e 74 anos e de oito anos em $53 \%$ no grupo daqueles com idade $>75$ anos. Concluíram que a revascularizaçāo do miocárdio bem sucedida entre os pacientes idosos confere alívio consistente da angina e longevidade que excede a da população dos Estados Unidos da América, quando equiparada para sexo e idade.

Com esta observação parcial da literatura, podemos constatar as alternativas disponíveis e os resultados obtidos a curto e médio prazos, quando se trata cirurgicamente a população dos octogenários.

O objetivo deste estudo foi levantar os dados relativos aos pacientes octogenários necropsiados no InCór entre janeiro de 1978 e dezembro de 1990 , e extrair informaçōes visando melhorar a qualidade e/ou a expectativa de vida deste segmento populacional, em nosso meio.

GRÁFICO 1

\section{Sistemas Desencadeantes do Óbito}

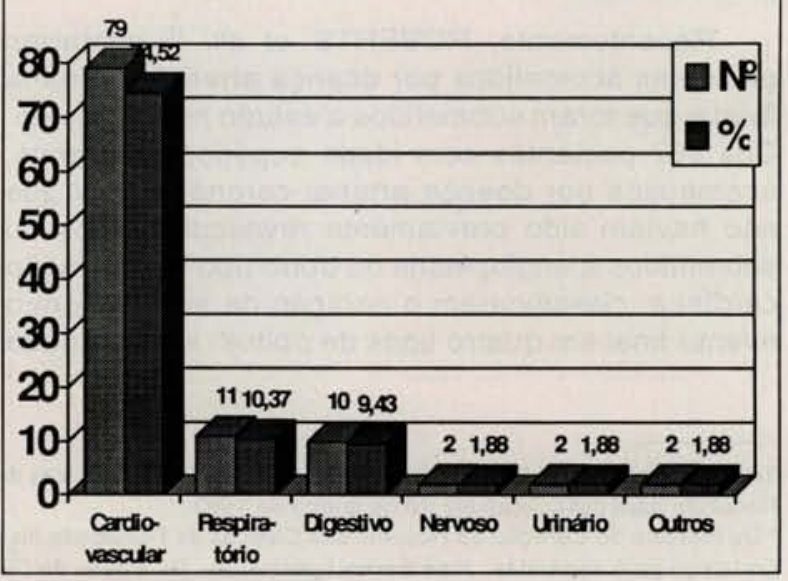


IGLÉZIAS, J. C. R.; LOURENÇĀO Jr., A.; DALLAN, L. A.; GUTIERRES, P. S.; RAMIRES, J. A. F.; STOLF, N. A. G.; OLIVEIRA, S. A.; VERGINELLI, G.; JATENE, A. D. - Octogenários: resultados de 3659 necropsias (12 anos de observaçāo). Rev. Bras. Cir. Cardiovasc., 8(1):39-43, 1993.

\section{CASUÍSTICA, MÉTODOS E RESULTADOS}

No InCór, entre janeiro de 1978 e dezembro de 1990, foram realizadas 3659 necropsias. Destas, 106 eram relativas a pacientes com idade $\geqslant 80$ anos, sendo $55(51,88 \%)$ do sexo masculino e 51 $(48,11 \%)$ do sexo feminino. A média de idade foi de 83,66 ( 80 a 93) anos. Os dados foram coletados, cruzados, analisados, sem que nenhum tratamento estatístico específico tenha sido aplicado.

Dentre os sintomas e sinais, encontramos, em ordem decrescente de freqüência, dor precordial em $43(40,56 \%)$ pacientes, dispnéia em $27(25,47 \%)$, síncope em $9(8,49 \%)$, confusăo mental em 7 $(6,60 \%)$, dor abidominal em $5(4,71 \%)$, dor torácica em $4(3,77 \%)$, prostraçāo em $2(1,88 \%)$, melena em $2(1,88 \%)$, lombalgia em $2(1,88 \%)$, edema de membros inferiores em $1(0,94 \%)$, tosse em $1(0,94 \%)$, hematêmese em $1(0,94 \%)$, prostatismo em 1 $(0,94 \%)$ e diarréia em $1(0,94 \%)$ (Gráfico 3). Os sintomas haviam se iniciado há mais de seis meses em $56(52,85 \%)$ pacientes, enquanto, nos outros 50 $(47,16 \%)$, há menos de seis meses.

Dentre os sintomas orgânicos classificados como desencadeantes do óbito, aparecem, em ordem decrescente de freqüência, os seguintes: cardiovascular em $79(74,52 \%)$ pacientes, respiratório em $11(10,37 \%)$, digestivo em $10(9,43 \%)$, nervoso em

GRÁFICO 2

\section{Causas Cardiovasculares do Cóbito}

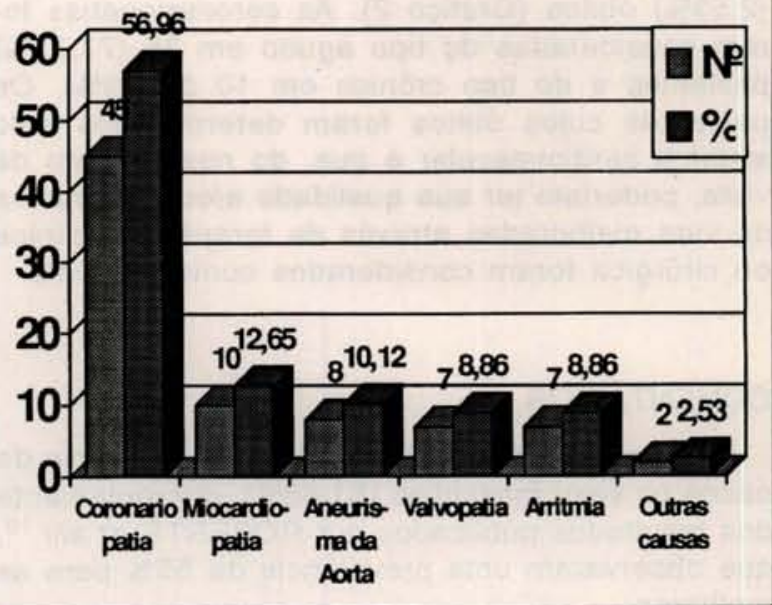

GRÁFICO 3

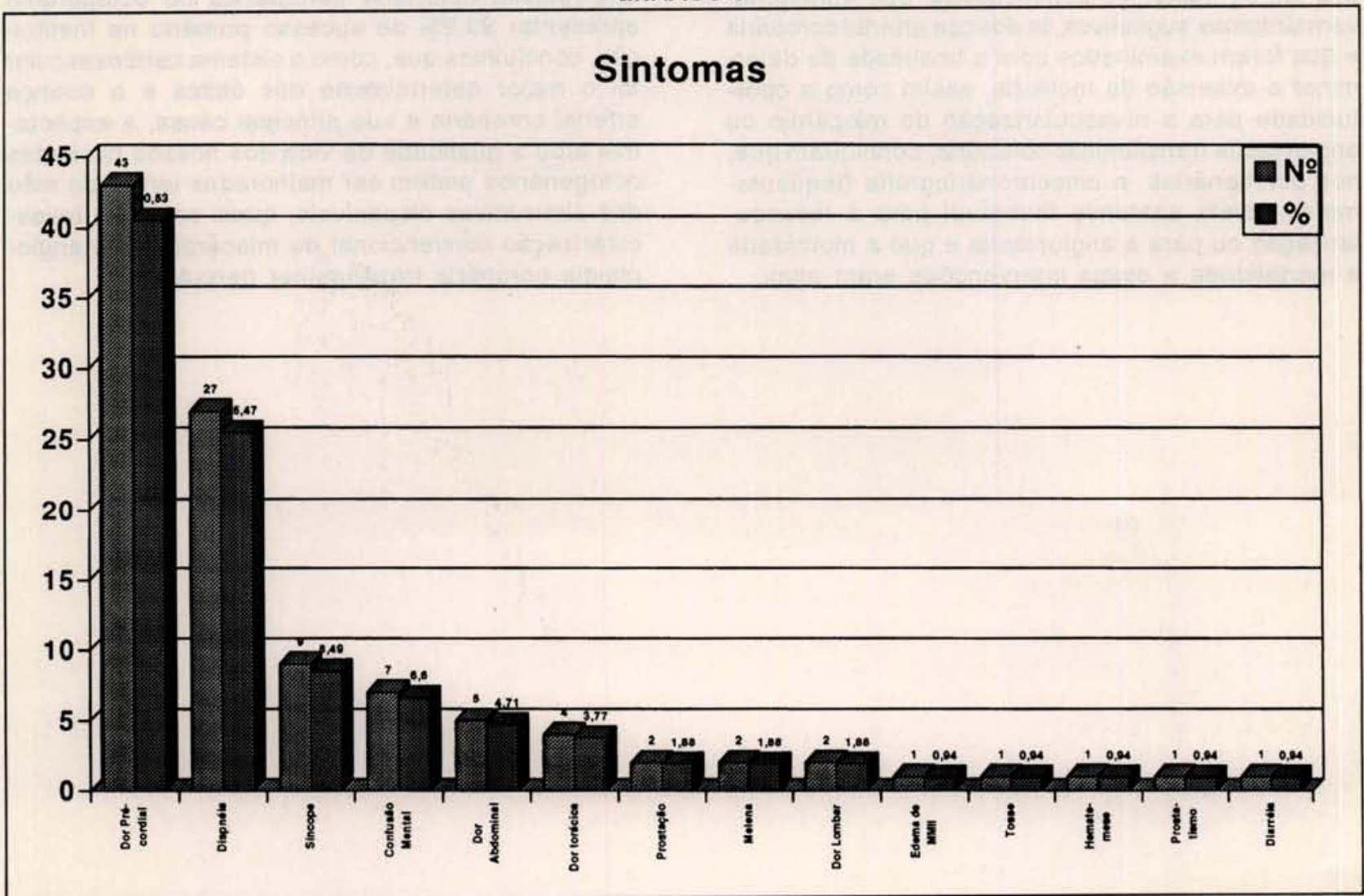


IGLÉZIAS, J. C. R.; LOURENÇĀO Jr., A.; DALLAN, L. A.; GUTIERRES, P. S.; RAMIRES, J. A. F.; STOLF, N. A. G.; OLIVEIRA, S. A.; VERGINELLI, G.; JATENE, A. D. - Octogenários: resultados de 3659 necropsias (12 anos de observaçāo). Rev. Bras. Cir. Cardiovasc., 8(1):39-43, 1993.

$2(1,88 \%)$, urinário em $2(1,88 \%)$; outros eventos foram considerados como desencadeantes do óbito em $2(1,88 \%)$ pacientes (Gráfico 1$)$. Considerando o sistema cardiovascular, verificamos que a doença arterial coronária foi a determinante do óbito em $45(56,96 \%)$ pacientes, a miocardiopatia em 10 $(12,65 \%)$, os aneurismas da aorta em $8(10,12 \%)$, as valvopatias em $7(8,86 \%)$, as arritmias em 7 $(8,86 \%)$ e outras causas foram responsáveis por 2 $(2,53 \%)$ óbitos (Gráfico 2). As coronariopatias foram consideradas do tipo agudo em $35(77,77 \%)$ pacientes e do tipo crônico em $10(22,23 \%)$. Os pacientes cujos óbitos foram determinados pelo sistema cardiovascular e que, do nosso ponto de vista, poderiam ter sua qualidade e/ou expectativa de vida melhoradas através de terapêutica clínica ou cirúrgica foram considerados como tratáveis.

\section{COMENTÁRIOS}

Em nossa casuística, houve um predomínio de óbitos no sexo masculino $(51,88 \%)$, diferentemente dos resultados publicados por ROBERTS et alii ${ }^{10}$, que observaram uma prevalência de $55 \%$ para as mulheres.

Concordamos com KOWALCHUK et alii ${ }^{5}$, quando analisando os aspectos angiográficos encontrados em 84 pacientes consecutivos, que apresentavam sintomas sugestivos de doença arterial coronária e que foram examinados com a finalidade de determinar a extensāo da moléstia, assim como a oportunidade para a revascularização do miocárdio ou angioplastia transluminar coronária, concluíram que, nos octogenários, a cinecoronariografia freqüentemente revela anatomia favorável para a revascularizaçāo ou para a angioplastia e que a morbidade e mortalidade a essas intervençōes eram altas.
Estamos de acordo com ARONOW et alii ${ }^{1} \mathrm{e}$ $\mathrm{COHN} \& \mathrm{JOHNSON}^{3}$, quando afirmam que a fraçāo de ejeção do ventrículo esquerdo representa uma das variáveis mais importantes para o prognóstico de sobrevida do octogenário revascularizado, e MULLANY et alii ${ }^{8}$, quando afirmam que a revascularização do miocárdio para o octogenário, embora associada com risco operatório aumentado, propicia um excelente alívio dos sintomas e boa sobrevida de cinco anos; representa, portanto, uma alternativa válida para os pacientes.

Dentre os fatores previsores para a elevada mortalidade hospitalar dos pacientes idosos submetidos a revascularização, citados por LOOP et alii 6 (idade > 75 anos, tabagismo, prejuízo da função ventricular esquerda e sexo feminino), não houve correspondência em nossa casuística com o sexo.

Considerando que: 1) 79 (74,52\%) dos óbitos desta casuística foram determinados pelo sistema cardiovascular; 2) dos anteriores, $45(56,96 \%)$ determinados por coronariopatias; 3 ) o início da sintomatologia ter ocorrido num período maior que seis meses em $50(47,16 \%)$ pacientes; 4) a revascularizaçāo cirúrgica convencional do miocárdio ter mortalidade hospitalar atual de $8,5 \%{ }^{4}$ na Instituição; 5) a mortalidade hospitalar nos anos de 1991, 1992 e janeiro de 1993 ter sido igual a zero; 6) a angioplastia coronária percutânea no octogenário apresentar $93,2 \%$ de sucesso primário na Instituição, concluímos que, como o sistema cardiovascular foi o maior determinante dos óbitos e a doença arterial coronária a sua principal causa, a expectativa e/ou a qualidade de vida dos nossos pacientes octogenários podem ser melhoradas lançando mão das alternativas disponiveis, quais sejam: a revascularização convencional do miocárdio ou a angioplastia coronária transluminar percutânea. 
IGLÉZIAS, J. C. R.; LOURENÇĀO Jr., A.; DALLAN, L. A.; GUTIERRES, P. S.; RAMIRES, J. A. F.; STOLF, N. A. G.; OLIVEIRA, S. A.; VERGINELLI, G.; JATENE, A. D. - Octogenários: resultados de 3659 necropsias (12 anos de observação). Rev. Bras. Cir. Cardiovasc., 8(1):39-43, 1993.

RBCCV 44205-197

IGLÉZIAS, J. C. R.; LOURENÇĀO Jr., A.; DALLAN, L. A.; GUTIERRES, P. S.; RAMIRES, J. A. F.; STOLF, N. A. G.; OLIVEIRA, S. A.; VERGINELLI, G.; JATENE, A. D. - Octagenarians: results of 3659 necropsies. Rev. Bras. Cir. Cardiovasc., 8(1):39-43, 1993.

ABSTRACT: Between January 1978 and December 1990, 3659 autopsies were performed in our Institution, of which 106 of the patients were $\geqslant 80$ years old. Of these patients, $55(51.88 \%)$ were male and $51(48.11 \%)$ female, and the mean age was 83.66 ( 80 to 93 ) years old. The main symptoms presented prior to death were: angina in $40.56 \%$, dyspnea in $25.47 \% \%$, and syncope in $8.49 \%$ of the patients. In $47.16 \%$ the symptoms had begun more than six months before. The systems related to the cause of death were found to be: cardiovascular in $74.52 \%$, respiratory in $10.37 \%$, digestive in $9.43 \%$, nervous in $1.88 \%$, urinary in $1.88 \%$ and others in $1.88 \%$. Among the cardiovascular deaths: $56.96 \%$ were due to coronary artery disease, $12.65 \%$ due to myocardiopathy, $10.12 \%$ due to thoracic aortic aneurysm and $8.86 \%$ were atributed to arrythmias. It was considered that: the cardiovascular system was the major determinant of death in $74.52 \%$, coronary disease was responsible for $56.96 \%$ of the cardiovascular deaths, standard revascularization in this group has a mortality rate of $8.5 \%$ and that primary angioplasty has had a success rate of $93.2 \%$. It was therefore concluded that the patients of at least 80 years old should be maintained near their physician or the time between their medical check-up be reduced in order to significantly improve their quality and expectancy of life.

DESCRIPTORS: necropsies, elderly patients.

\section{REFERÊNCIAS BIBLIOGRÁFICAS}

1 ARONOW, W. S.; AHN, C.; KRONZON, I. - Prognosis of congestive heart failure in elderly patients with normal versus abnormal left ventricular systolic function associated with coronary artery disease. Am. J. Cardiol., 66:1257-1259, 1990.

2 CHAITMAN, B. R.; RYAN, T. J.; KRONWAL, R. A.; FORSTER, E. D.; FROMMER, P. L.; KILLIP, T. Coronary artery surgery study (CASS). Comparability of 10 year survival in randomized and randomizable patients. J. Am. Coll. Cardiol., 16:1071-1078, 1990.

3 COHN, J. N. \& JOHNSON, G. and Veterans Administration Cooperative Study Group. - Heart failure with normal ejection fraction: the V-Heft study. Circulation, 8 (Suppl. 3):48-53, 1990.

4 IGLÉZIAS, J. C. R.; DALLAN, L. A. O.; OLIVEIRA, S. F.; RAMIRES, J. A. F.; OLIVEIRA, S. A.; VERGINELLI, G.; PILEGGI, F.; JATENE, A. D. Revascularizaçāo do miocárdio no paciente octogenário. Rev. Bras. Cir. Cardiovsc., 5:183-186, 1990.

5 KOWALCHUK, G. J.; SIU, S. C.; LEWIS, S. M. Coronary artery disease in the octogenarian: angiographic spectrum and suitability for revascularization. Am. J. Crdiol., 66:1319-1323, 1990.

6 LOOP, F. D.; LYTLE, B. W.; COSGROVE, D. M.; GOORMASTIC, M.; TAYLOR, P. C.; GOLDING, L. A. R.; STEWART, R. W.; GILL, C. C. - Coronary artery bypass graft surgery in the elderly. Cleveland
Clin. J. Med., 55:23-34, 1988.

7 MACAYA, C.; ALFONSO, F.; INIGUEZ, A.; ZARCO, P. - Long term clinical and angiographic follow-up of percutaneous transluminal coronary angioplasty in patients greater than or equal to 65 yers of age. Am. J. Cardiol., 66:1513-1515, 1990.

8 MULLANY, C.J.; DARLING, G. E.; PLUTH, J. R.; ORSZULAK, T. A.; SCHAFF, H. V.; ILSTRUP, D. M.; GERSH, B. J. - Early and late results after isolated coronary artery bypass surgery in 159 patients aged 80 years and older. Circulation, 82 (Supl. 4):229-236, 1990

9 RENKIN, J.; MELIN, J.; ROBERT, A.; RICHELLE, F.; BACHY, J. L.; COP, J.; DETRY, J. M.; WIJNS, W. - Detection of restenosis after successful coronary angioplasty: improved clinical decision making with use of logistic model combining procedural and followup variables. J. Am. Coll. Cardiol., 16:1333-1340, 1990.

10 ROBERTS, W. C.; KRAGEL, A. H.; POTKIN, B. N. Ages at death and sex distribution in age decade in fatal coronary artery disease. Am. J. Cardiol., 66:1379-1381, 1990 .

11 WEINTRAUB, W. S.; COHEN, C. L.; CURLING, P. E.; JONES, E. L.; CRAVER, J. M.; GUYTON, R.; KING III, S. B.; DOUGLAS, J. S. - Results of coronary surgery after failed elective coronary angioplasty in patients with prior coronary surgery. J. Am. Coll. Cardiol., 16:1341-1347, 1990. 